\title{
Growth and characterization of a new chelating agent added 4-dimethylamino- $N$-methyl-4-stilbazolium tosylate (DAST) single crystals
}

\author{
A.S. Haja Hameed ${ }^{\text {a }}$, S. Rohani ${ }^{\text {a }}$, W.C. Yu ${ }^{\text {b }}$, C.Y. Tai ${ }^{\text {c }}$, C.W. Lan ${ }^{\text {c,* }}$ \\ ${ }^{a}$ Department of Chemical and Biochemical Engineering, The University of Western Ontario, London, Ontario, Canada N6A 5B9 \\ ${ }^{\mathrm{b}}$ Department of Molecular Science and Engineering, National Taipei University of Technology, Taipei 106, Taiwan \\ ${ }^{\mathrm{c}}$ Department of Chemical Engineering, National Taiwan University, Taipei 10617, Taiwan \\ Received 9 January 2006; received in revised form 9 October 2006; accepted 2 November 2006
}

\begin{abstract}
A new chelating agent diethylenetriaminepentaacetic acid (DTPA) is employed for the first time to investigate its effect on the metastable zone width, crystal growth and characterization of DAST single crystals. The width of DAST in methanol is measured with and without adding DTPA and EDTA. The presence of DTPA in the DAST solutions enhances the metastable zone width as compared to the EDTA added solutions. The pure and the chelating agents added DAST crystals are grown by the slow cooling method and their crystal size distributions are presented. The addition of the chelating agent DTPA significantly suppresses spurious nucleation and cluster formation in the DAST solution, so that fewer, but larger crystals are easily obtained. The crystalline powder of the grown crystals is examined by X-ray diffraction. The Fourier transform infrared (FTIR) and UV-vis-NIR spectroscopic analyses have been done for the DAST samples. The hardness of the grown pure, DTPA and EDTA added DAST crystals is measured on the $\left(\begin{array}{lll}0 & 0 & 1\end{array}\right)$ face under different loads.
\end{abstract}

(C) 2006 Elsevier B.V. All rights reserved.

PACS: 81.10.Dn; 81.70.Pg

Keywords: Metastable zone width; Nucleation; Solubility; Growth from solution; Recrystallization; Organic compounds; Nonlinear optical materials

\section{Introduction}

Among the many well known nonlinear optical (NLO) materials, the organic ionic salt crystal 4- $\mathrm{N}, \mathrm{N}$-dimethylamino$4^{\prime}-\mathrm{N}$-methyl-stilbazolium tosylate (DAST) is a very promising NLO material, which has been demonstrated to have a very large NLO susceptibility and the largest electro-optic (EO) coefficient of all materials researched up to date [1-5]. It belongs to the monoclinic crystal system with the noncentro symmetric space group $C c$ and the point group $m$. It has the following lattice parameters: $a=10.365 \AA, b=11.322 \AA, c=17.893 \AA, Z=4$ and $\beta=92.24^{\circ}[1]$.

Although DAST crystals have shown the excellent NLO properties in many applications, the growth of high-quality large crystals has been a challenging task. Pan et al. and Sohma et al.

\footnotetext{
* Corresponding author. Tel.: +88622363 3917; fax: +88622363 3917. E-mail address: cwlan@ntu.edu.tw (C.W. Lan).
}

$[6,7]$ have illustrated the feasibility of the growth of large single crystals more than $16 \mathrm{~mm}$ in diameter by seeded growth. Recently, we have grown $2 \mathrm{~cm}$ size DAST crystals by the two zone growth technique [8]. Even though high quality DAST crystals were grown by the step nucleation method (SNM), the number of nuclei is still difficult to control. Among the several methods to manipulate crystal nucleation behavior and further controlling crystal growth, the use of a chelating agent, such as ethylenediaminetetraacetic acid (EDTA) could be useful in nucleation control as well. Indeed, several successful examples have been obtained for both organic and inorganic crystals [9-13].

In search of new chelating agents, we have found that diethylenetriaminepentaacetic acid (DTPA) is a suitable chelating agent to suppress the formation of nuclei and clusters in the case of DAST solution. It is a colorless and white powder. This polycarboxylic multidentate ligand has an excellent chelating ability compared to other hexadentate ligands like EDTA. DTPA binds to a number of metals like $\mathrm{Zn}^{2+}, \mathrm{Mg}^{2+}, \mathrm{Mn}^{2+}$, 
<smiles>COC(=O)CCN(CCCN(CC(=O)[O-])CC(=O)[O-])CC(=O)[O-]</smiles>

EDTA Ethylenediam in etetraacetic acid anion<smiles>COC(=O)CN(CCC(=O)[O-])CCN(CCN(CC(=O)[O-])CC(=O)[O-])CC(=O)[O-]</smiles>

Diethylenetriaminepentaacetic acid anion

Fig. 1. Chemical structures of EDTA and DTPA anions.

$\mathrm{Co}^{2+}, \mathrm{Cu}^{2+}, \mathrm{Pb}^{2+}, \mathrm{Ca}^{2+}$ and various radio-nuclides. DTPA has five acetate moieties linked by a molecular backbone (Fig. 1) which can tightly complex a metallic ion and forms more stable complexes than that made by other chelating agents like EDTA. It is structurally similar to EDTA except that it has an extra basic unit of $\mathrm{NCH}_{2} \mathrm{COO}^{-}$and diethylene in place of ethylene in EDTA. The chemical structures of EDTA and DTPA are shown in Fig. 1. Since DTPA is stronger than EDTA, it is often used to lower the effective free metal content of processes. Compared to EDTA, DTPA forms more stable metal complexes, which are more resistant to oxidation and are more compatible in concentrated electrolytes, liquid alkali, etc. As per the above statements, DTPA is expected to be more effective in controlling the nucleation rate and suppressing the cluster formation by capturing metallic ions in the solution. It has been found that the incorporation of DTPA in the DAST solution enhances the zone width significantly compared to the pure and EDTA added solutions. Also it is observed that it reduces the number of spurious nucleation and enhances the growth rate of the crystals.

In this present study, the metastable zone width was measured for pure, DTPA, EDTA added DAST solutions. Their crystal size distributions were analyzed. The grown crystals were characterized by the XRD, FTIR and UV-vis-NIR spectroscopic analyses. The hardness of the grown crystals was estimated by the Vickers micro hardness studies.

\section{Experimental}

DAST was synthesized by the condensation of 4-methyl- $N$-methyl pyridinium tosylate and 4- $N, N$-dimethylamino-benzaldehyde in the presence of piperidine $[8,14,15]$. 4-Methyl- $N$-methyl pyridinium tosylate used in the condensation reaction was prepared from equimolar quantities of 4-picoline and methyl- $p$-toluenesulphonate. The entire synthesis process was conducted in dry nitrogen atmosphere to avoid the formation of the orange hydrated form of DAST. The resulting DAST material was further purified by recrystallization from methanol. The recrystallized DAST crystals were used for the nucleation and crystal growth experiments.

For all the experiments, 20,30 and $40 \mathrm{ppm}$ of EDTA and DTPA were used. The nucleation experiments were carried out in small cells $(20 \mathrm{ml})$ with and without the addition of DTPA and EDTA. The advantages of the small experiment have been described in ref. [15]. The small-cell experiments were carried out by putting growth cells into a tray and immersing the tray in a constanttemperature bath (CTB) for nucleation and crystal growth experiments. The accuracy of CTB is within $\pm 0.01{ }^{\circ} \mathrm{C}$. In each cell, to avoid the sticking of the

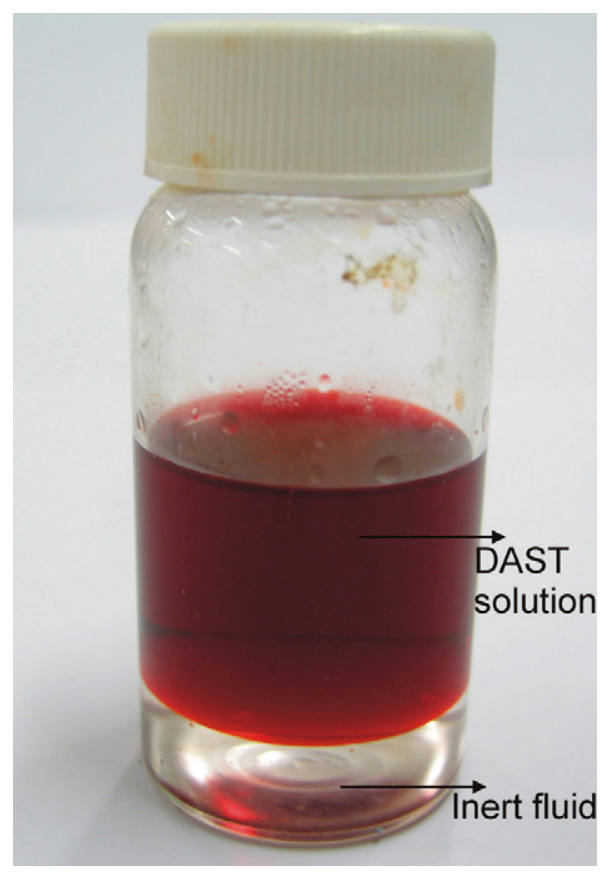

Fig. 2. A clear view of inert fluid-DAST solution interface.

crystals on the glass wall, an inert fluid, 3 M Fluorinert electronic liquid FC-77, was used to let the DAST solution sit; $5 \mathrm{ml}$ of preheated FC-77 inert fluid and $10 \mathrm{ml}$ of DAST solution were added for each cell. The clear interface between the inert fluid and the DAST solution was very helpful in the observation of nucleation (Fig. 2). In addition, FC-77 did not wet DAST crystals and hence it helped significantly in harvesting the DAST crystals grown while keeping the solvent from sticking to their surface. To start the nucleation experiments, the solutions were kept at $50^{\circ} \mathrm{C}$ for 2 days for homogenization and then cooled to the saturation temperature of $40^{\circ} \mathrm{C}$ at a cooling rate of $1{ }^{\circ} \mathrm{Ch}^{-1}$. The cooling rate was reduced to $1^{\circ} \mathrm{C} \mathrm{day}^{-1}$ from the saturation temperature. Metastable zone width was measured by the conventional poly-thermal method [16,17]. In this method, the equilibrium saturated solution was cooled from the overheated temperature until a visible crystal was observed.

In order to check the consistency of the results from the nucleation experiments, four experiments for each case were carried out under the same condition.

By means of a diffractometer (MAC Science Diffractometer MXP-3), X-ray diffraction pattern was recorded for the powdered DAST samples in the range of $5-40^{\circ}$ where the monochromatic wavelength $1.5418 \AA(\mathrm{Cu} \mathrm{K} \alpha)$ was used. In order to check the incorporation of DTPA or EDTA in the grown DAST crystals, a FTIR spectrometer (Perkin-Elmer, Spectrum One) was used to record the spectrum the range of $600-2000 \mathrm{~cm}^{-1}$ for the corresponding DAST crystalline powders. Using a UV-vis spectrometer (Model: Jasco 570, Japan), the crystal transmission ranging from the visible to near-IR region was measured. Vickers hardness measurements were done on $(001)$ face of the grown crystals using a micro-hardness tester (Model HMV-2, Shimadzu, Japan). Five indentations were made on each sample for each load. The diagonal lengths of each indentation were recorded and the averages of the diagonal lengths were used for calculations. The microhardness value of a particular sample was taken by averaging the different values of microhardness at various loads where near constancy in the value of hardness is achieved. At least three crystals from each case were used for the reproducibility.

\section{Results and discussion}

\subsection{Metastable zone width of pure, DTPA and EDTA added DAST solutions}

From the solubility studies, no significant change in solubility was observed in the additives added DAST solutions because the 

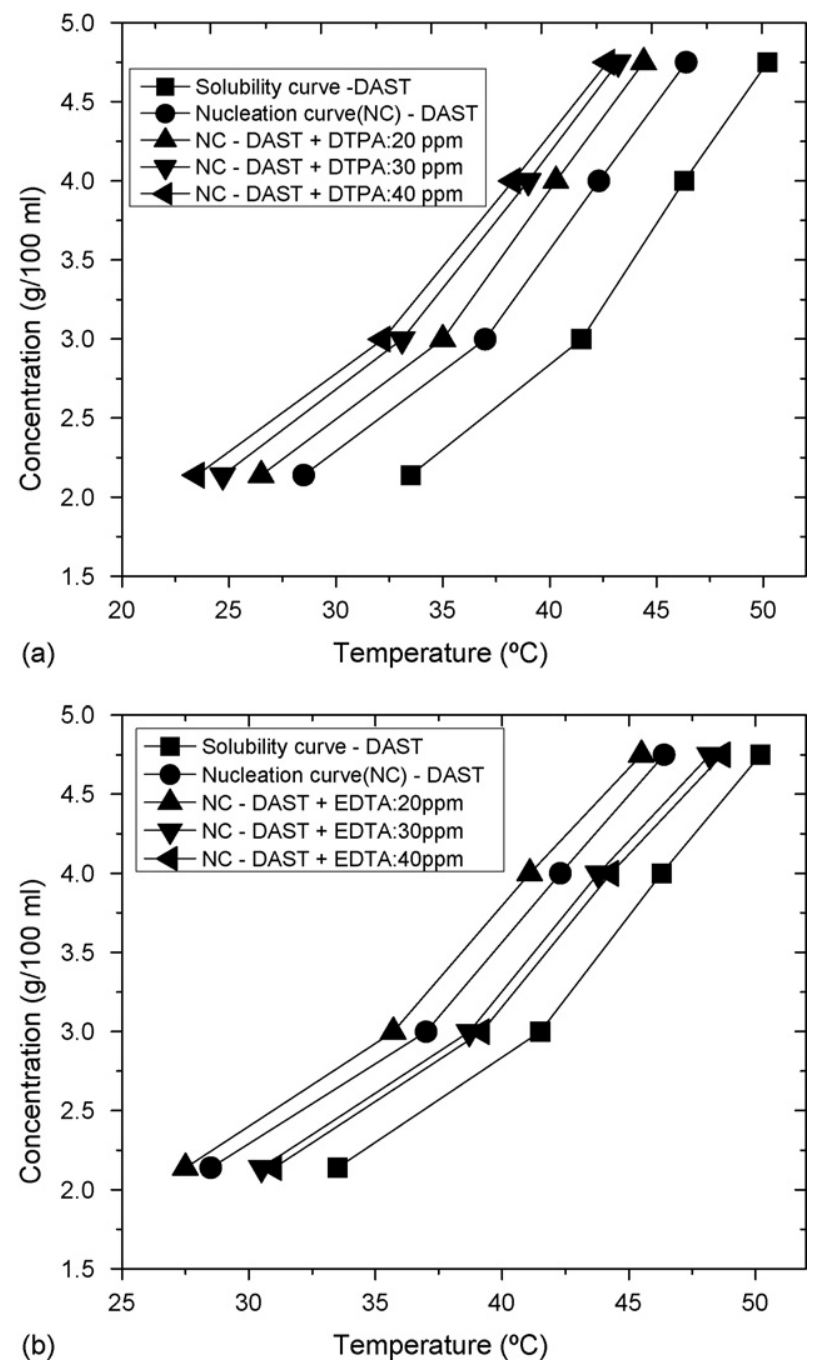

Fig. 3. Metastable zone width of (a) pure and DTPA added DAST solutions and (b) EDTA added DAST solutions.

amount of the chelating agent added was very small (20, 30 and $40 \mathrm{ppm})$. The solubility of DAST reported in the present paper was consistent with the data given by Wu et al [15]. In ref. [15], the metastable zone widths of the DAST solutions prepared at a saturation temperature of $40^{\circ} \mathrm{C}$ were about 4,6 and $7^{\circ} \mathrm{C}$ for the pure ( 0 ppm EDTA), 10 and 20 ppm EDTA added solutions, respectively. The metastable zone widths (MZW) of pure, DTPA and EDTA added DAST solutions as a function of temperature are shown in Fig. 3. From the figure, the metastable zone widths of the DAST solutions saturated at $41.5^{\circ} \mathrm{C}$ were about $4.5,5.8$, 2.8 and $2.3{ }^{\circ} \mathrm{C}$ for the pure (0 ppm EDTA), 20, 30 and $40 \mathrm{ppm}$ EDTA added solutions, respectively. Only a small variation in the metastable stable zone was observed as compared to the reported values. But in general, the addition of EDTA (up to $20 \mathrm{ppm}$ ) enhanced the metastable zone width in both reports. Here, it should be mentioned that the metastable zone width was affected by a number of factors, such as stirring rate, cooling rate of the solution and presence of additional crystals or impurities [18-21]. In the present study, $40 \mathrm{ppm}$ of DTPA was found to be the threshold concentration in the DAST solutions, at which the maximum zone width was observed, after this limit, the DAST solutions remained unstable.

The results obtained from the metastable zone width experiments showed that the addition of DTPA enhanced the zone width. Pure DAST solution had the MZW of $5^{\circ} \mathrm{C}$. The MZW of 20,30 and 40 ppm DTPA added DAST solutions were 7, 8.8 and $10{ }^{\circ} \mathrm{C}$, respectively. The metastable zone width was increased by the addition of DTPA. In the case of EDTA added DAST, the zone width was $5.8^{\circ} \mathrm{C}$ for $20 \mathrm{ppm}$ EDTA added DAST solution. For 30 and $40 \mathrm{ppm}$ EDTA added solutions, the metastable zone widths decreased abruptly and were about 2.8 and $2.3^{\circ} \mathrm{C}$ for the respective solutions at the lower saturation temperature.

In ref. [15], it has been reported that $20 \mathrm{ppm}$ seemed to be the maximum amount of EDTA that could be added for the experiments and too much of EDTA added caused the precipitation of EDTA crystals on the DAST crystals leading to poor surface quality. The same situation was again observed in the present work. Hence $20 \mathrm{ppm}$ EDTA added DAST solution showed an increased metastable zone width but as its concentration was increased, the width was found to be decreased abruptly. This trend was not observed in the case of DTPA added DAST solutions. If the concentration of DTPA was increased, the zone width also increased. Since DTPA was found to be more soluble than EDTA in DAST solution, the precipitation of DTPA crystals was not observed whereas in the case of EDTA added DAST solutions, the precipitation of EDTA crystals was found. Also, it was believed that DTPA at higher concentration levels bonded strongly with the metallic ions rather than EDTA due to polycarboxylic multidentate ligands, which lead to an excellent chelating ability compared to other hexadentate ligands like EDTA. According the above reasons, the zone width was an erratic way in the EDTA added DAST solutions but it regularly increased in the case of DTPA added DAST solutions. It was believed that the enhancement of metastable zone width in all the DTPA added solutions used in the present work was mainly due to the reduction of chemical activity due to the strong chelating action of DTPA.

\subsection{Crystal growth and crystal size distribution}

For the crystal growth of pure, DTPA or EDTA added DAST, 20, 30 and $40 \mathrm{ppm}$ of DTPA or EDTA were added in the DAST solutions. The DAST crystals grown at the different concentrations of DTPA and EDTA are shown in Fig. 4. The harvested crystals were classified according to their sizes, and the size distributions are given in Fig. 5. Since there were many tiny crystals in the pure and EDTA ( 30 and $40 \mathrm{ppm}$ ) added DAST solutions, their weights were used rather than number in expressing size distributions. The results of the experiments were consistent and the crystal photographs shown in Fig. 4 are from one of the experimental runs, for reference.

From the results of the crystal size distribution, DTPA (20, 30 and $40 \mathrm{ppm}$ ) interacted strongly rather than EDTA (up to $20 \mathrm{ppm}$ ) with DAST or impurity ions in the solution. The strong interaction between metal ions present in the solutions was dependent on many parameters such as the size of the metal ion, the ability of the chelating agent to fold, the number of 
(a)

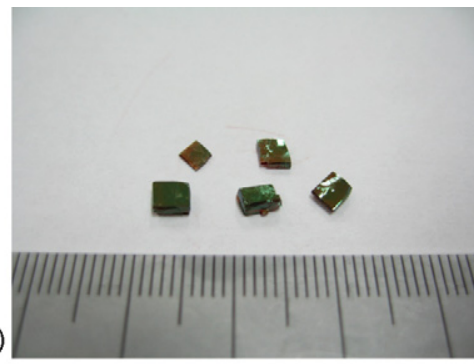

(d)

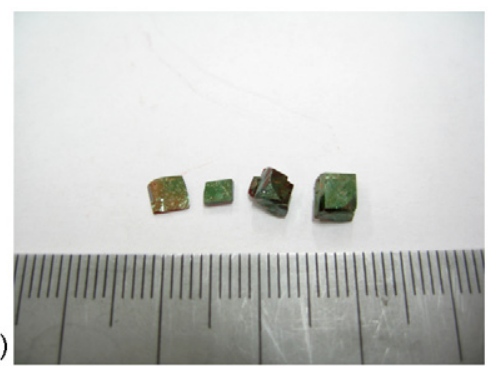

(b)

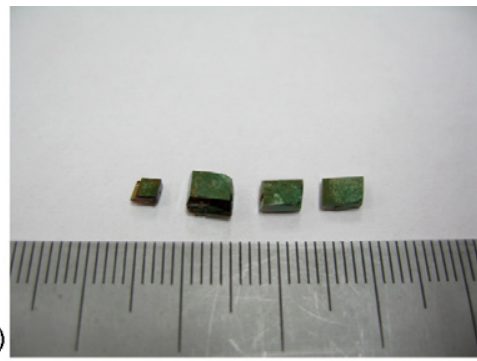

(e)

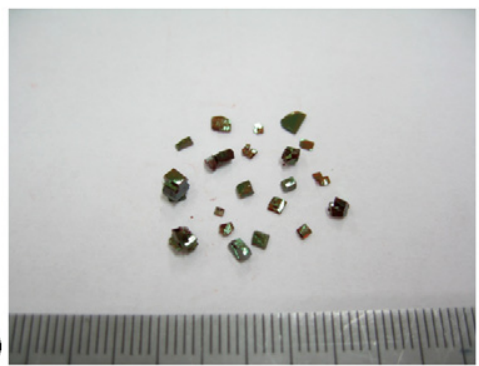

(f)
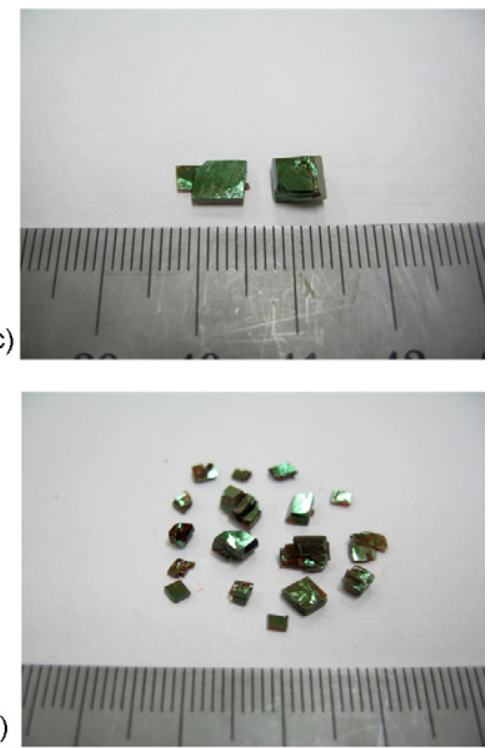

(g)

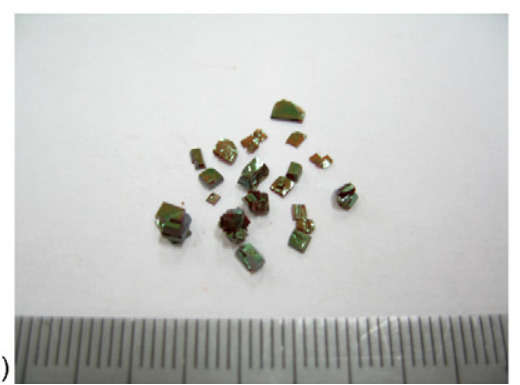

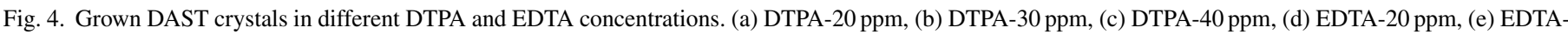
$30 \mathrm{ppm}$, (f) EDTA-40 ppm and (g) pure DAST.

interactions between the metal ion and the chelater and several subtle energetic co-ordination. Due to the excellent chelating ability of DTPA, the rate of crystallization was reduced in the DTPA added solutions. Thus, only a few crystals were obtained

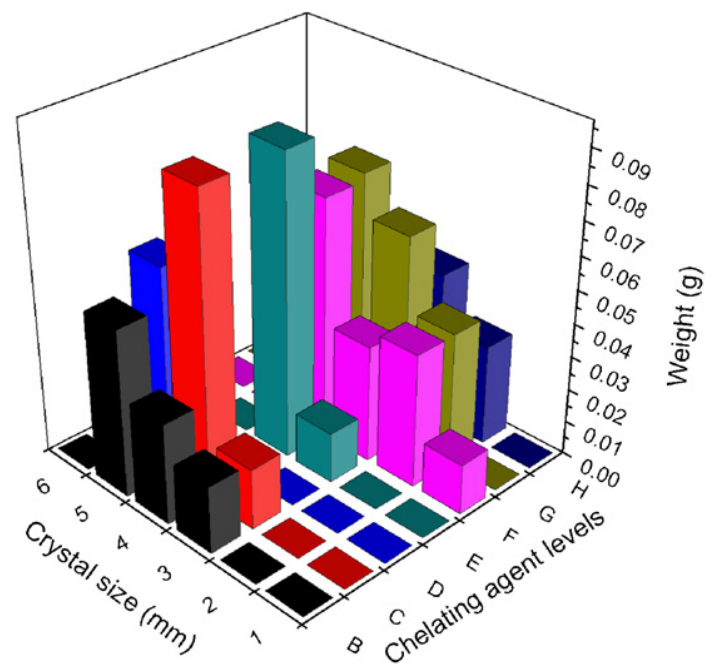

Fig. 5. The crystal size distribution of pure, DTPA and EDTA added DAST. (B) DAST + DTPA-20 ppm, (C) DAST + DTPA-30 ppm, (D) DAST + DTPA$40 \mathrm{ppm}, \quad$ (E) DAST + EDTA-20 ppm, (F) DAST + EDTA-30 ppm, (G) DAST + EDTA-40 ppm and $(\mathrm{H})$ pure DAST. from 20 ppm of EDTA and DTPA added DAST solutions. This controlled crystallization became more probable with increasing DTPA concentration. But it was improbable with the increasing EDTA concentration in the DAST solution. The positive active parts (more in DTPA than EDTA) engaged strongly to capture the metal ions in the DTPA added solutions. Many heterogeneous nucleations were observed in the EDTA (except $20 \mathrm{ppm}$ ) added DAST solutions where a forced induced nucleation was prompted due to the precipitation of EDTA crystals, which are acting as nucleation sites. Hence many tiny crystals were obtained from the more EDTA added DAST solutions.

With the addition of DTPA, the clustering phenomenon was markedly suppressed. Therefore, in fact, DTPA improved crystal size and decreased the number of crystals. The addition of 30 and 40 ppm of DTPA into the DAST solution reduced clustering to a great extent which in turn yielded bigger size crystals. As shown in Fig. 4(c), in 40 ppm DTPA added DAST solution, only two DAST crystals were obtained.

The addition of DTPA reduced the formation of spurious nucleation at 30 and 40 ppm levels, whereas in the case of EDTA at 30 and 40 ppm levels, there were many tiny crystals. The presence of more EDTA in the solutions caused the precipitation of EDTA crystals not only on DAST crystal surface (which leaded to poor surface quality) but also on the inert fluid-DAST solution interface. The obtained two crystals from 40 ppm DTPA added DAST solutions had the size of $6 \mathrm{~mm}$ each along $a$ and $b$ 
Table 1

Lattice parameter values of pure, DTPA and EDTA added DAST crystals

\begin{tabular}{llll}
\hline Samples & \multicolumn{3}{l}{ Lattice parameter values $(\AA)$} \\
\cline { 2 - 4 } & $a$ & $b$ & $c$ \\
\hline Pure DAST & 10.366 & 11.323 & 17.891 \\
DAST:DTPA-20 ppm & 10.367 & 11.320 & 17.892 \\
DAST:DTPA-30 ppm & 10.365 & 11.321 & 17.893 \\
DAST:DTPA-40 ppm & 10.366 & 11.321 & 17.893 \\
DAST:EDTA-20 ppm & 10.367 & 11.319 & 17.895 \\
DAST:EDTA-30 ppm & 10.366 & 11.320 & 17.896 \\
DAST:EDTA-40 ppm & 10.368 & 11.320 & 17.897 \\
\hline
\end{tabular}

axes. Moreover, 20 ppm EDTA added DAST solution gave good results in terms of crystal growth (Fig. 4(d)) i.e. it controlled the nucleation rate as well.

From the above studies, the number of nucleation decreased in the DTPA added DAST solutions. Moreover, the surface morphology of the crystals was considerably improved and the clustering of crystals was significantly reduced. It could be observed from the results that the effect of chelaters in suppressing spurious nucleation and clustering was more significant in 30 and $40 \mathrm{ppm}$ of DTPA, and $20 \mathrm{ppm}$ of EDTA added solutions. The crystal growth in $20 \mathrm{ppm}$ of EDTA added DAST solution was consistent with the previous report [15]. The enhancement of metastable regime increased the stability of the solution and hence bigger size crystals could be harvested from the DTPA added DAST solutions at higher concentration levels.

\subsection{Characterization studies}

From the X-ray powder diffraction patterns of pure, DTPA, EDTA added DAST samples (Fig. 6), the lattice parameter values were calculated using $2 \theta$ values of high intensity peaks corresponding to the $h \mathrm{kl}$ planes using the monoclinic crystallographic equation. The lattice parameter values of the grown crystals are shown in Table 1. The calculated lattice parameter values of pure, DTPA and EDTA added DAST crystals were comparable with that of pure one and with the values reported by Marder et al. [1]. The small variations in the values of lattice parameter $c$ were observed in the case of more EDTA added DAST crystals. This variation was not observed in the DTPA added DAST crystals.

The FTIR spectra of pure, DTPA and EDTA added DAST samples are shown in Fig. 7. From the spectra, there was considerable absorption observed in 30 and $40 \mathrm{ppm}$ of EDTA added DAST samples. At 1160 and $1180 \mathrm{~cm}^{-1}$ plane aromatic ring deformations, which are typical for para-substituted benzenes were observed. The $\mathrm{CH}_{3}$ umbrella deformation was assigned to $1347 \mathrm{~cm}^{-1}$. In-plane stretching frequency of $\mathrm{C}-\mathrm{C}=\mathrm{C}-\mathrm{C}$ was found at $1577 \mathrm{~cm}^{-1}$. There was no incorporation of DTPA or EDTA in the DAST molecular structure, which was revealed from the FTIR analysis.

The optical absorption spectra of $40 \mathrm{ppm}$ EDTA and $40 \mathrm{ppm}$ DTPA added DAST crystals are shown in Fig. 8. The absorption of pure and EDTA added DAST crystals are reported earlier [15]. From the observed UV-vis-NIR spectra, it was found that
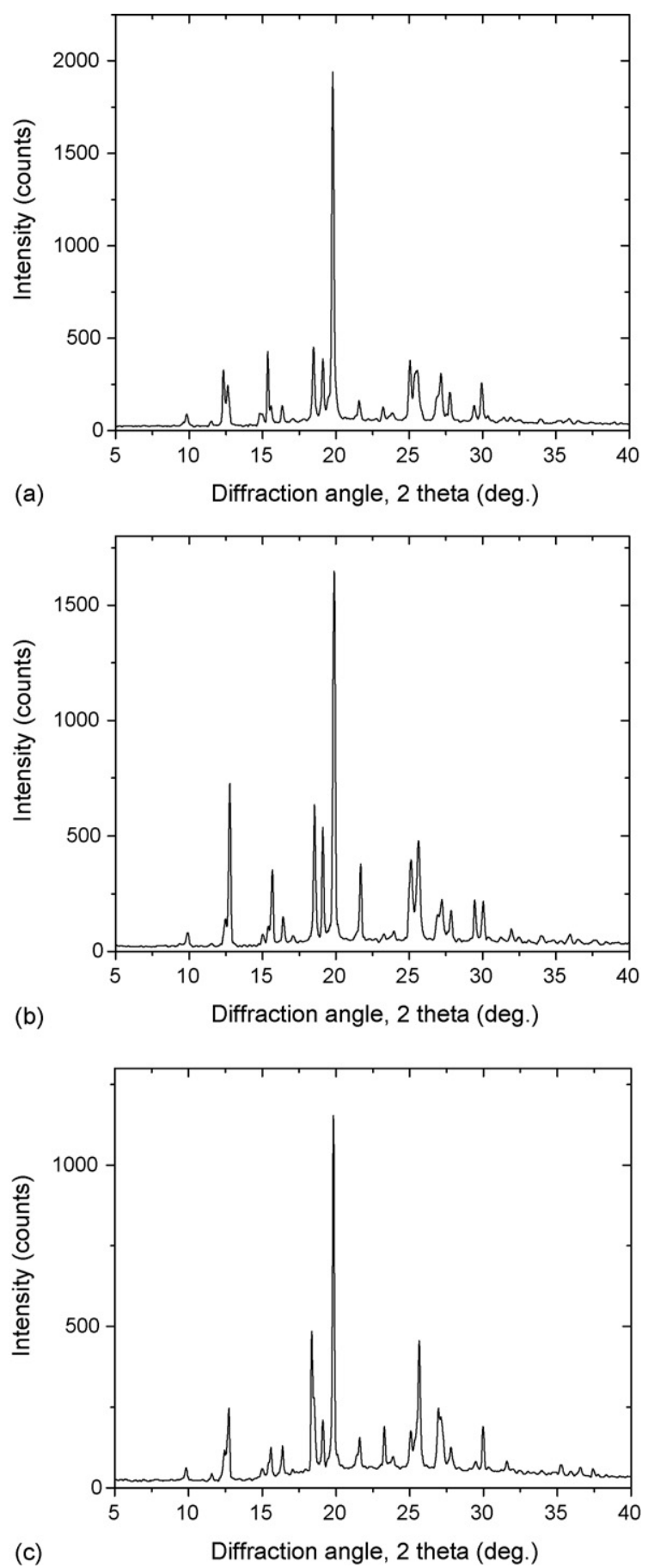

Fig. 6. X-ray diffraction pattern of (a) pure, (b) $30 \mathrm{ppm}$ DTPA added DAST and (c) 30 ppm EDTA added DAST.

40 ppm DTPA added DAST crystal had about $30 \%$ of absorption, whereas $40 \mathrm{ppm}$ EDTA added DAST crystals exhibited a more absorption, which was about $70 \%$. The difference in the absorptions was mainly due to the following reasons. During crystal growth, an effective suppression of chemical activity of metal ions occurred in the DTPA added DAST solutions due to the formation of complexes with the functional groups of DTPA by its chelating action. Since more metal ions captured by DTPA than EDTA in the solutions, there was a slower nucleation rate and low cluster formation in the DTPA added solutions during 


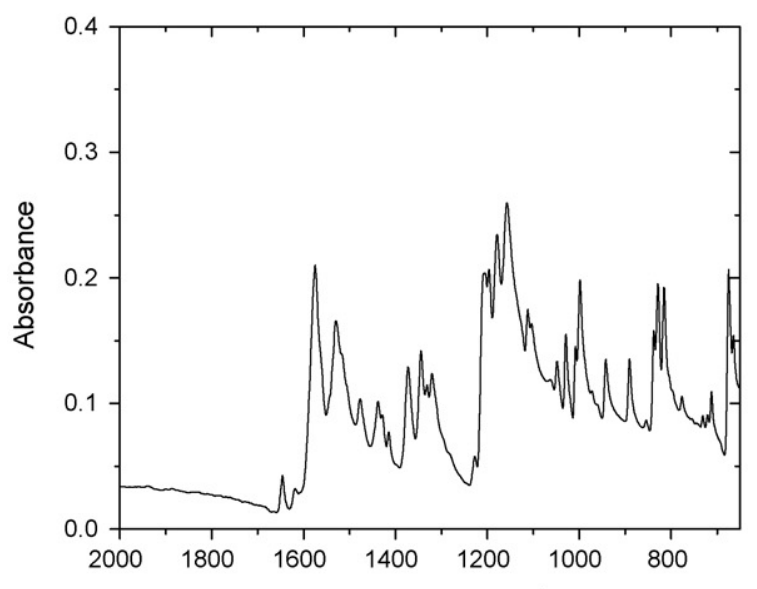

(a)

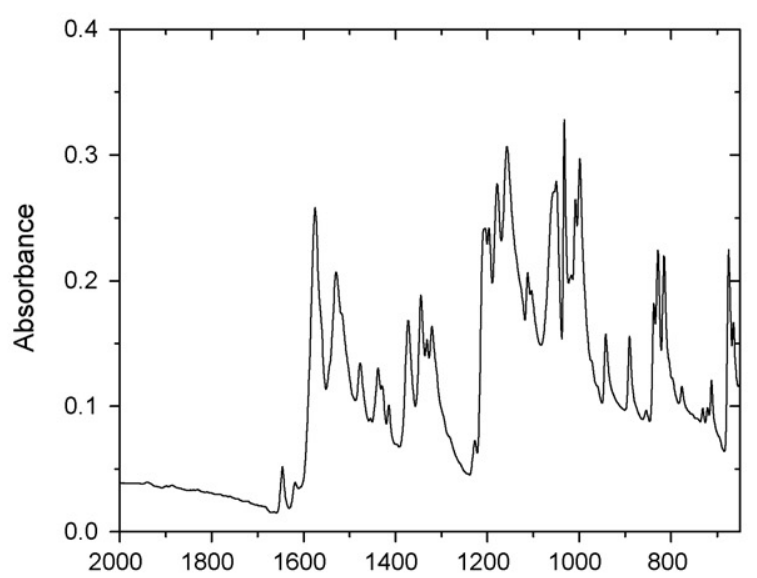

(b)

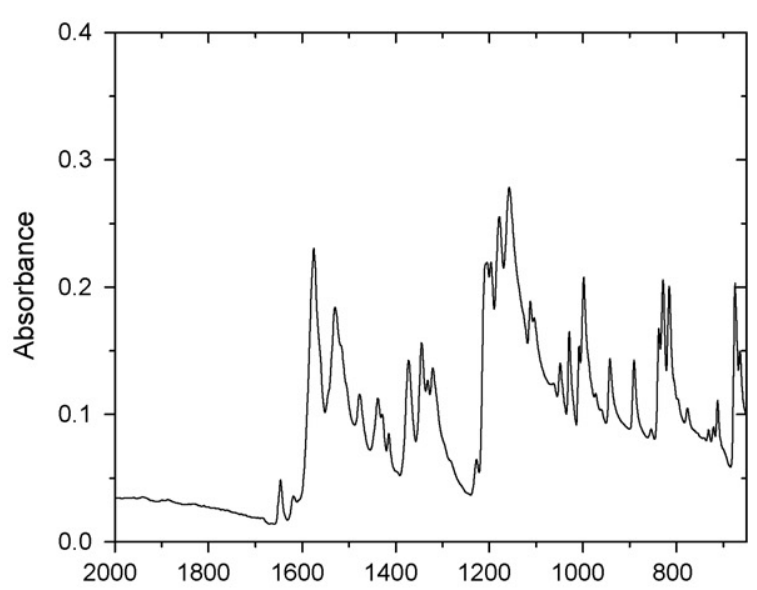

(c)

Wavenumber $\left(\mathrm{cm}^{-1}\right)$

Fig. 7. FTIR spectra of (a) pure, (b) 40 ppm DTPA added DAST and (c) 40 ppm EDTA added DAST.

the crystal growth. Therefore good quality crystals with smooth surface were grown. But a reverse trend was observed in the EDTA added DAST crystals due to low quality and rough surface originating from the precipitation of EDTA crystals on the surface of DAST crystals. The absorption edge was observed at about $700 \mathrm{~nm}$, which was $100 \mathrm{~nm}$ right shift with the previous report [15]. The change in the absorption edge was observed
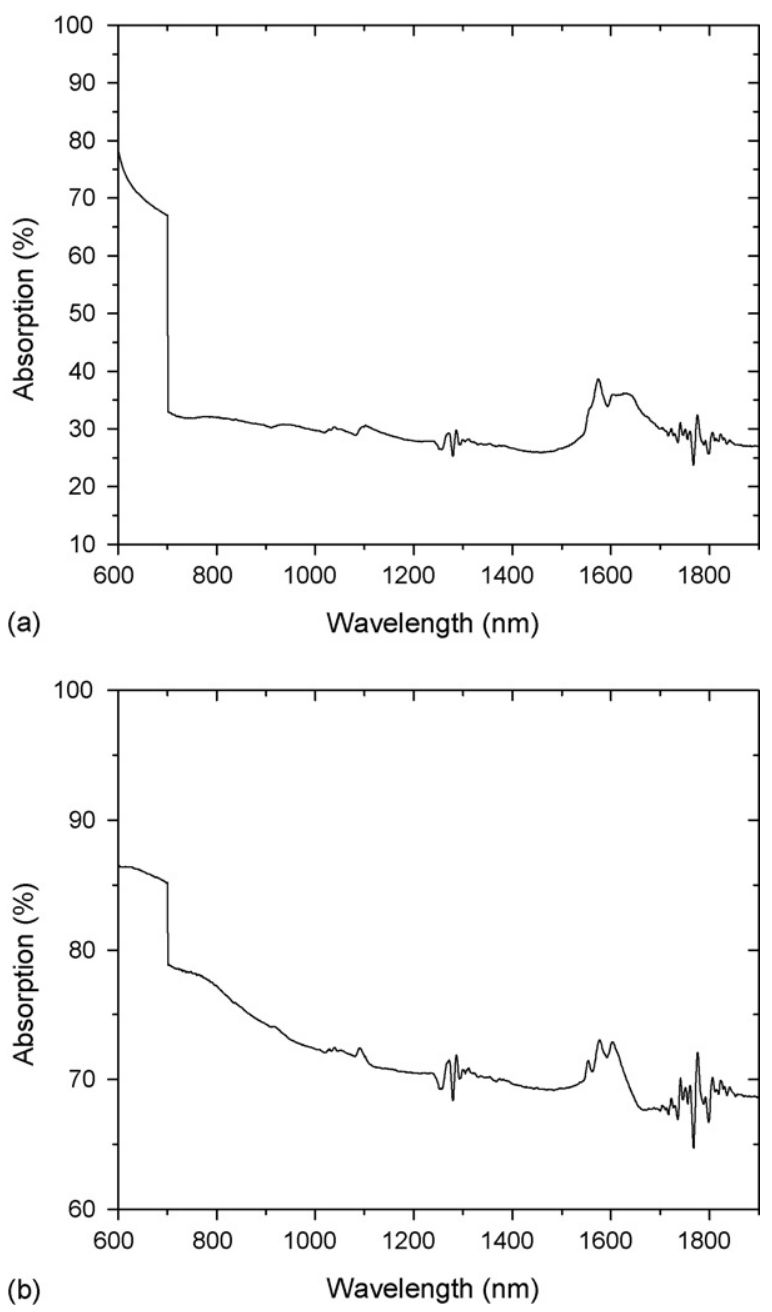

Fig. 8. Absorption spectra of as grown (a) $40 \mathrm{ppm}$ DTPA added DAST and (b) 40 ppm EDTA added DAST crystals.

in pure, DTPA and EDTA added DAST crystals. The variation was mainly due to the orientation of the cationic chromophore in DAST crystal lattice with respect to its dielectric axes.

The microhardness was measured on the $\left(\begin{array}{lll}0 & 0 & 1\end{array}\right)$ face for pure, DTPA and EDTA added DAST crystals under different loads. The average diagonal length of the indented impression was calculated. If the $P$ is the applied load (in $\mathrm{kg}$ ) and $d$ is the average diagonal length of the indentation impressions $(\mathrm{mm})$, and the angle between the opposite faces of the diamond pyramid is $\theta=136^{\circ}$, then $H_{\mathrm{v}}$ in $\mathrm{kg} \mathrm{mm}^{-2}$ is given by

$H_{\mathrm{v}}=\frac{1.8544 P}{d^{2}}$

The variation of microhardness $\left(H_{\mathrm{v}}\right)$ with the applied loads for pure, DTPA and EDTA added DAST crystals are shown in Fig. 9. From the figure, the decrease of $H_{\mathrm{v}}$ with the increase of applied load was observed. It was due to the fact that as energy associated with the indenter was low at lower load $(5 \mathrm{~g})$, dislocation at the point of indentation did not allow the indenter to penetrate further. But at higher load, the indenter could easily penetrate owing to the movement of dislocations away from the indenter. 


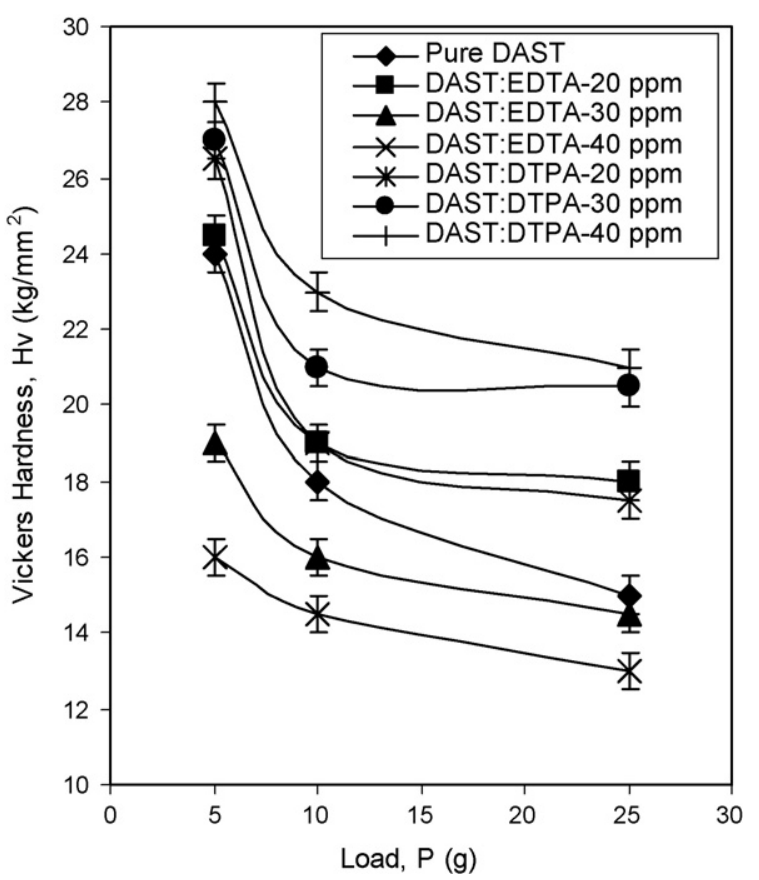

Fig. 9. Variation of microhardness with the different loads for pure, DTPA and EDTA added DAST crystals.

The microhardness values of pure DAST crystals were approximately 24,18 and $15 \mathrm{~kg} \mathrm{~mm}^{-2}$ for the loads of 5,10 and $25 \mathrm{~g}$, respectively. The microhardness number was found in the range of $21-28 \mathrm{~kg} \mathrm{~mm}^{-2}$ for the DTPA added DAST crystals. In the case of EDTA added DAST crystals, the values were estimated in the range of $13-19 \mathrm{~kg} \mathrm{~mm}^{-2}$. From the figure, with increasing additive concentration, $H_{\mathrm{v}}$ increased in the DTPA added DAST crystals and decreased in the EDTA added DAST crystals. The reasons for the increased hardness values in the DTPA added DAST crystals are explained as follows. DTPA (20, 30 and 40 ppm) interacted strongly than EDTA (except $20 \mathrm{ppm}$ ) with DAST or impurity ions in the solution. Since DTPA captured more metal ion than EDTA, an effective suppression of chemical activity of metal ions occurred in the DTPA added DAST solutions. Also, EDTA (except 20 ppm) added DAST crystals possessed the reduced hardness values due to low crystal quality and rough $(001)$ face resulted from the precipitation of EDTA crystals on the surface of DAST crystals.

\section{Conclusions}

Diethylenetriaminepentaacetic acid (DTPA) was found to be a new chelating agent to enhance the meta-stability and nucleation control in DAST solution. It can be used for the crystal growth of several organic or inorganic materials, that have lim- itable metastable zone widths in their appropriate solvents. In the present study, the metastable zone width of DAST solutions was found with the different concentrations of DTPA and EDTA. It was found that the incorporation of DTPA in the DAST solution enhanced the zone width significantly compared to the pure and more EDTA added solutions. It could be found that the incorporation of DTPA reduced the number of spurious nucleation, clustering of crystals and it enhanced the growth rate of the crystals as well. The DAST crystals grown at $20 \mathrm{ppm}$ of DTPA and EDTA gave the almost same results in terms of crystal growth. From the X-ray powder diffraction study, the lattice parameters were determined for the DAST samples. From the FTIR spectra, it was observed that there was no incorporation of DTPA or EDTA in the DAST molecular structure. The absorption of the grown crystals was measured. The microhardness studies revealed that the addition of DTPA increased the hardness of the DAST crystals.

\section{References}

[1] S.R. Marder, J.W. Perry, W.P. Schaefer, Science 245 (1989) 626.

[2] C.P. Yakymyshyn, K.R. Stewart, E.P. Boden, S.R. Marder, J.W. Perry, P. Schaefer, Proceedings of the Second International Symposium on Organic Materials for Non-linear Optics, Royal Society of Chemistry, Cambridge, UK, 1991, p. 108.

[3] G. Knofple, R. Schlesser, R. Ducret, P. Gunter, Nonlinear Opt. 9 (1995) 143.

[4] F. Pan, G. Kno"fple, Ch. Bosshard, S. Follonier, R. Spreiter, M.S. Wong, P. Gunter, Appl. Phys. Lett. 69 (1996) 13.

[5] Ch. Bosshard, R. Spreiter, P. Gunter, J. Opt. Soc. Am. B 18 (2001) 1620.

[6] F. Pan, M.S. Wong, Ch. Bosshard, P. Gunter, Adv. Mater. 8 (1996) 592.

[7] S. Sohma, H. Takahashi, T. Taniuchi, H. Ito, Chem. Phys. 245 (1999) 359.

[8] A.S. Haja Hameed, W.C. Yu, Z.B. Chen, C.Y. Tai, C.W. Lan, J. Crystal Growth 282 (2005) 117.

[9] F.J. Welcher, The Analytical Use of EDTA, D. Van Nostrand Company, Canada, 1957.

[10] K. Srinivasan, K. Meera, P. Ramasamy, J. Cryst. Growth 205 (1999) 457.

[11] K. Srinivasan, K. Meera, P. Ramasamy, Cryst. Res. Technol. 3 (2000) 291.

[12] N.P. Rajesh, K. Meera, K. Srinivasan, P. Santhana Raghavan, P. Ramasamy, J. Cryst. Growth 213 (2000) 389.

[13] N.P. Rajesh, P. Santhana Raghavan, P. Ramasamy, C.W. Lan, J. Chin. Inst. Chem. Eng. 33 (2002) 325.

[14] H. Adachi, Y. Takahashi, J. Yabuzaki, Y. Mori, T. Sasaki, J. Cryst. Growth 198-199 (1999) 568.

[15] J.I. Wu, R. Gopalakrishnan, C.I.D. Tai, C.W. Lan, Jpn. J. Appl. Phys. 43 (2004) 1507.

[16] J. Nyvlt, O. Sohnel, J.J. Zola, G. Kostescky, J. Cryst. Growth 62 (1983) 543.

[17] N.Zaitseva, L.N. Rashkovich, S.V. Bogatyreva, J. Cryst. Growth 148 (1995) 276.

[18] J.W. Mullin, Crystallization, 2nd ed., Butterworths, London, 1971.

[19] J. Nyvlt, J. Cryst. Growth 3-4 (1968) 377.

[20] J. Nyvlt, R. Rychly, J. Gottfried, J. Wurzelova, J. Cryst. Growth 6 (1970) 151.

[21] J. Nyvlt, Industrial Crystallization from Solutions, Butterworths, London, 1972. 